\title{
Convergence Analysis of Discrete Differential Geometry Operators over Surfaces
}

\author{
Zhiqiang $\mathrm{Xu}^{1}$, Guoliang $\mathrm{Xu}^{2}$, and Jia-Guang $\mathrm{Sun}^{3}$ \\ 1 Department of Computer Science, Tsinghua University, Beijing 100084, China \\ xuzq@tsinghua.edu.cn \\ 2 ICMSEC, LSEC, Academy of Mathematics and System Sciences, \\ Chinese Academy of Sciences, Beijing, China \\ xuguo@lsec.cc.ac.cn \\ 3 School of Software, Tsinghua University, Beijing 100084, China \\ sunjg@tsinghua.edu.cn
}

\begin{abstract}
In this paper, we study the convergence property of several discrete schemes of the surface normal. We show that the arithmetic mean, area-weighted averaging, and angle-weighted averaging schemes have quadratic convergence rate for a special triangulation scenario of the surfaces. By constructing a counterexample, we also show that it is impossible to find a discrete scheme of normals that has quadratic convergence rate over any triangulated surface and hence give a negative answer for the open question raised by D.S.Meek and D.J. Walton. Moreover, we point out that one cannot build a discrete scheme for Gaussian curvature, mean curvature and Laplace-Beltrami operator that converges over any triangulated surface.
\end{abstract}

\section{Introduction}

Estimation of normal vectors and curvatures on discrete surfaces are often required in Computer Aided Geometric Design and Computer Graphics. In the past decades, many discretized approaches for normal vectors, Gaussian curvature, mean curvature and Laplace-Beltrami operator have been proposed and used. The convergence of the discretized approaches has also been studied. In [5], the authors analyzed the convergence of the normal vector and Gaussian curvature. For normal vectors, they obtained the following result: for non-uniform data, the unit vector parallel to the arithmetic mean of unit normals of the triangular faces around a point approximates the unit normal of the surface at that point to accuracy $O(h)$. Furthermore, by the numerical test, they found that the accuracy of the arithmetic mean, area-weighted averaging, and angle-weighted averaging are not higher than $O(h)$. As pointed out in [5], normal estimation methods with accuracy $O\left(h^{2}\right)$ are very useful for the spherical image method of Gaussian curvature approximation. Hence, they raised an open question:find a linear combination of the normals of the triangular faces, based on geometric considerations, that approximates the normal of the surface to $O\left(h^{2}\right)$. In this paper, we prove that under certain conditions, the approximation accuracy 
of normal vectors can be $O\left(h^{2}\right)$, meaning the approximation converges with a quadratic rate. Moreover, we show that it is impossible to find a discretization scheme of normals that has quadratic convergence rate over any triangulated surface. Hence, the answer to the above mentioned open question is negative.

In 6], Meyer et al. proposed some discrete schemes to approximate several important geometric attributes, including normal vectors and curvatures on arbitrary triangular meshes. In [9], G. Xu proved that a well known discretized scheme of Gaussian curvature, derived from Gauss-Bonnet theorem, has quadratic convergence rate under certain conditions. In [10] and 11], he also studied the convergence of Laplace-Beltrami operators and mean curvature, include Taubin et al's discretization 7, Mayer et al's discretization 4, Desbrun et al's discretization [1, Meyer et al 's discretization [6, and proposed several simple discretization schemes of Laplace-Beltrami operator over triangulated surfaces. In [5], the author proposes an asymptotic analysis of Gaussian curvature for three methods: quadratic fit method, angular defect and spherical image method. A review of these schemes is given in [3. However, none of these discretizations of Gaussian curvature and mean curvature has been proved to be convergent over any non-degenerate triangulated surface. Therefore, a natural questions is raised: can one build a discrete scheme of Gaussian curvature and mean curvature which involves one-ring vertices and converges over any non-degenerate triangle surface? In this paper, we shall give a negative answer to this question. Hence, we have to accept the fact that the discretization scheme for curvature only convergent over special triangular surface.

The rest of the paper is organized as follows. In Section 2, we introduce some definitions and formulations. In Section 3, we discuss the convergence property of discrete schemes of normals. In Section 4, by giving a counterexample, we show that one cannot construct a scheme of Gaussian curvature and mean curvature that converges over any non-degenerate triangle surface. Moreover, we also give a negative answer to the open question raised in [5].

\section{Preliminaries}

Let $\mathbf{S}(u, v)=(x(u, v), y(u, v), z(u, v)) \in \mathbf{R}^{3}$ be a regular parametric surface. We further assume that the point where the normal and curvature need to be approximated is $\mathbf{O}:(x(0,0), y(0,0), z(0,0))^{T}$. Then from differential geometry, the normal vector of $\mathbf{S}(u, u)$ at $\mathbf{O}$ is $\mathbf{S}_{\mathbf{u}}(\mathbf{0}, \mathbf{0}) \times \mathbf{S}_{\mathbf{v}}(\mathbf{0}, \mathbf{0})$.

Let $\mathbf{P}_{i}=\mathbf{S}\left(\mathbf{q}_{i}\right)$ be $n$ distinct points on $\mathbf{S}(x, y)$ near the point $(x(0,0), y(0,0)$, $z(0,0))^{T}$ and $\mathbf{q}_{i}=\left(r_{i} \cos \left(\theta_{i}\right) h, r_{i} \sin \left(\theta_{i}\right) h\right)$. The indices arithmetic modulo $n$ so index $n+1$ is the same as index 1 . Without loss of generality, we assume $0 \leq \theta_{1} \leq \theta_{2} \leq \cdots \leq \theta_{n}<2 \pi$. Denote the normal to the triangle $\mathbf{P}_{i} \mathbf{O} \mathbf{P}_{i+1}$ as $\mathbf{n}_{i, i+1}$, by using Taylor expansion,

$$
\begin{aligned}
\mathbf{n}_{i, i+1}= & \left(\mathbf{P}_{i}-\mathbf{O}\right) \times\left(\mathbf{P}_{i+1}-\mathbf{O}\right) \\
= & \left(\begin{array}{c}
\left(y_{u} z_{v}-y_{v} z_{u}\right) \sin \left(\theta_{i+1}-\theta_{i}\right) r_{i} r_{i+1} h^{2}+A_{i} h^{3}+O\left(h^{4}\right) \\
-\left(\left(x_{u} z_{v}-x_{v} z_{u}\right) \sin \left(\theta_{i+1}-\theta_{i}\right) r_{i} r_{i+1} h^{2}+B_{i} h^{3}+O\left(h^{4}\right)\right) \\
\left(x_{u} y_{v}-x_{v} y_{u}\right) \sin \left(\theta_{i+1}-\theta_{i}\right) r_{i} r_{i+1} h^{2}+C_{i} h^{3}+O\left(h^{4}\right)
\end{array}\right),
\end{aligned}
$$


where

$$
\begin{aligned}
A_{i} & =\left(y_{u u} \cos ^{2} \theta_{i}+2 y_{u v} \cos \theta_{i} \sin \theta_{i}+y_{v v} \sin ^{2} \theta_{i}\right)\left(z_{u} \cos \theta_{i+1}+z_{v} \sin \theta_{i+1}\right) r_{i}^{2} r_{i+1} \\
& -\left(z_{u u} \cos ^{2} \theta_{i}+2 z_{u v} \cos \theta_{i} \sin \theta_{i}+z_{v v} \sin ^{2} \theta_{i}\right)\left(y_{u} \cos \theta_{i+1}+y_{v} \sin \theta_{i+1}\right) r_{i} r_{i+1}^{2}, \\
B_{i} & =\left(x_{u u} \cos ^{2} \theta_{i}+2 x_{u v} \cos \theta_{i} \sin \theta_{i}+x_{v v} \sin ^{2} \theta_{i}\right)\left(z_{u} \cos \theta_{i+1}+z_{v} \sin \theta_{i+1}\right) r_{i}^{2} r_{i+1} \\
& -\left(z_{u u} \cos ^{2} \theta_{i}+2 z_{u v} \cos \theta_{i} \sin \theta_{i}+z_{v v} \sin ^{2} \theta_{i}\right)\left(x_{u} \cos \theta_{i+1}+x_{v} \sin \theta_{i+1}\right) r_{i} r_{i+1}^{2}, \\
C_{i} & =\left(x_{u u} \cos ^{2} \theta_{i}+2 x_{u v} \cos \theta_{i} \sin \theta_{i}+x_{v v} \sin ^{2} \theta_{i}\right)\left(y_{u} \cos \theta_{i+1}+y_{v} \sin \theta_{i+1}\right) r_{i}^{2} r_{i+1} \\
& -\left(x_{u u} \cos ^{2} \theta_{i}+2 x_{u v} \cos \theta_{i} \sin \theta_{i}+x_{v v} \sin ^{2} \theta_{i}\right)\left(y_{u} \cos \theta_{i+1}+y_{v} \sin \theta_{i+1}\right) r_{i} r_{i+1}^{2} .
\end{aligned}
$$

Denote the unit normal vector on triangle surfaces $\mathbf{P}_{i} \mathbf{O} \mathbf{P}_{i+1}$ as $\overline{\mathbf{n}}_{i, i+1}:=$ $\mathbf{n}_{i, i+1} /\left\|\mathbf{n}_{i, i+1}\right\|$. By using the formulation above, we have

$$
\overline{\mathbf{n}}_{i, i+1}=\overline{\mathbf{n}}_{0}\left(1-\frac{A_{i}\left(y_{u} z_{v}-y_{v} z_{u}\right) h-B_{i}\left(x_{u} z_{v}-x_{v} z_{u}\right) h+C_{i}\left(x_{u} y_{v}-x_{v} y_{u}\right) h}{\sin \left(\theta_{i+1}-\theta_{i}\right) r_{i} r_{i+1}}+O\left(h^{2}\right)\right) .
$$

where $\overline{\mathbf{n}}_{0}$ is the unit normal vector at $\mathbf{O}$, i.e.

$$
\overline{\mathbf{n}}_{0}=\frac{\left(y_{u} z_{v}-y_{v} z_{u},-\left(x_{u} z_{v}-x_{v} z_{u}\right), x_{u} y_{v}-x_{v} y_{u}\right)^{T}}{\sqrt{\left(y_{u} z_{v}-y_{v} z_{u}\right)^{2}+\left(x_{u} z_{v}-x_{v} z_{u}\right)^{2}+\left(x_{u} y_{v}-x_{v} y_{u}\right)^{2}}} .
$$

In general, the unit normal vector at $\mathbf{O}$ is approximated by

$$
\sum_{i=1}^{n} \lambda_{i} \overline{\mathbf{n}}_{i, i+1}
$$

where $\lambda_{i}$ is weight and $\sum_{i=1}^{n} \lambda_{i}=1$. By (2), we find the covergence rate of the discrete scheme is $O(h)$, which agrees with the result in [5].

There are several ways to determine the weights. A simple way is to take arithmetic mean, i.e., $\lambda_{i}=\frac{1}{n}$. Other ways include take an area-weighted average and an angle-weighted average.

Using discretization normals, the spherical image method for Gaussian curvature approximation is built in [5], and moreover, the following lemma is proved.

Lemma 1. (see [5]) When unit normals are known to accuracy $O\left(h^{2}\right)$, the spherical image method approximates the Gaussian curvature to accuracy $O(h)$.

Hence, $O\left(h^{2}\right)$ accuracy normals are very useful for computing the Gaussian curvature.

\section{Convergence of Normal Vectors}

In [5], the authors showed that the accuracy of the arithmetic mean, areaweighted averaging, and angle-weighted averaging are not higher than $O(h)$. 
However, we shall prove that under certain conditions, the approximation accuracy of the three ways can be $O\left(h^{2}\right)$.

We firstly exhibit the numerical behaviors of the discrete schemes of the surface normal. To show the numerical behavior of the discrete schemes, we take several two variable functions over xy-plane as three dimensional surfaces so that the exact normal can be computed. Both the exact and approximated normals are computed at some selected domain points $q_{i j}=\left(x_{i}, y_{j}\right)=(i / 20, j / 20), i=$ $1, \cdots, 19, j=1, \cdots, 19$. The surfaces are triangulated around $q_{i j}$ by triangulating the domain first, with mapping the planner triangulation onto the surfaces by the selected bivariate functions. As a simple case, the domain around $q_{i j}$ is triangulated locally by choosing $n$ regularly distributed points:

$$
q_{k}=q_{i j}+h\left(\cos \left(\theta_{k}\right), \sin \left(\theta_{k}\right)\right), \theta_{k}=2(k-1) \pi / n, k=1, \cdots, n .
$$

The convergence rate are checked by taking $h=1 / 8,1 / 16,1 / 32, \cdots$ and $n=$ $3,4, \cdots, 9$.

The functions we use are the following

$$
\begin{aligned}
& F_{1}(x, y)=\sqrt{4-(x-0.5)^{2}-(y-0.5)^{2}}, \\
& F_{2}(x, y)=\exp \left(-5\left((x-0.5)^{2}+(y-0.5)^{2}\right)\right), \\
& F_{3}(x, y)=\tan (5 y-5 x) \\
& F_{4}(x, y)=\frac{1+\cos (5 y)}{6+6(3 x-1)^{2}} .
\end{aligned}
$$

Denote $e_{1}\left(F_{j}, n\right), e_{2}\left(F_{j}, n\right)$ and $e_{3}\left(F_{j}, n\right)$ as the maximal error of the approximated surface normals computed by the arithmetic mean scheme,angle-weighted averaging and area-weighted averaging over the above mentioned local triangulations and the exact normal vector computed from the continuous surfaces defined by $F_{j}$. Tables $1-3$ show the asymptotic maximal error $e_{1}\left(F_{j}, n\right), e_{2}\left(F_{j}, n\right)$ and $e_{3}\left(F_{j}, n\right)$.

From the above numerical resuls, we find the arithmetic mean scheme and area-weighted averaging can converge in the rate $O\left(h^{2}\right)$ for the $n>3$ regularly distributed domain vertices. When the valence $\mathrm{n}$ is 3 , in general, the approximate surface normal converges in the rate $O(h)$. Moreover, if $n$ is even, the angleweighted averaging can converge in the rate $O\left(h^{2}\right)$.

Table 1. The maximal errors of the arithmetic mean scheme

\begin{tabular}{|c|c|c|c|c|}
\hline $\mathrm{n}$ & $e_{1}\left(F_{1}, n\right)$ & $e_{1}\left(F_{2}, n\right)$ & $e_{1}\left(F_{3}, n\right)$ & $e_{1}\left(F_{4}, n\right)$ \\
\hline 3 & $1.7291 e-02 \times h$ & $8.2968 e-01 \times h$ & $1.7331 e+00 \times h^{2}$ & $7.16229 e-01 \times h$ \\
\hline 4 & $7.2445 e-02 \times h^{2}$ & $5.4452 e-01 \times h^{2}$ & $1.1554 e+00 \times h^{2}$ & $6.3986 e-01 \times h^{2}$ \\
\hline 5 & $6.0409 e-02 \times h^{2}$ & $5.9247 e-01 \times h^{2}$ & $1.73312 e+00 \times h^{2}$ & $8.6673 e-01 \times h^{2}$ \\
\hline 6 & $5.6638 e-02 \times h^{2}$ & $6.6982 e-01 \times h^{2}$ & $1.73310 e+00 \times h^{2}$ & $9.0644 e-01 \times h^{2}$ \\
\hline 7 & $5.4428 e-02 \times h^{2}$ & $7.2413 e-01 \times h^{2}$ & $1.73310 e+00 \times h^{2}$ & $9.8637 e-01 \times h^{2}$ \\
\hline 8 & $5.3324 e-02 \times h^{2}$ & $7.6641 e-01 \times h^{2}$ & $1.73310 e+00 \times h^{2}$ & $1.0233 e-00 \times h^{2}$ \\
\hline 9 & $5.2663 e-02 \times h^{2}$ & $7.9757 e-01 \times h^{2}$ & $1.73310 e+00 \times h^{2}$ & $1.0458 e-00 \times h^{2}$ \\
\hline
\end{tabular}


Table 2. The maximal errors of the angle-weighted averaging

\begin{tabular}{|c|c|c|c|c|}
\hline $\mathrm{n}$ & $e_{2}\left(F_{1}, n\right)$ & $e_{2}\left(F_{2}, n\right)$ & $e_{2}\left(F_{3}, n\right)$ & $e_{2}\left(F_{4}, n\right)$ \\
\hline 3 & $3.0024 e-02 \times h$ & $7.7029 e-01 \times h$ & $2.1349 e+00 \times h^{2}$ & $7.1111 e-01 \times h$ \\
\hline 4 & $6.81814 e-02 \times h^{2}$ & $6.2178 e-01 \times h^{2}$ & $1.1560 e+00 \times h^{2}$ & $6.1403 e-01 \times h^{2}$ \\
\hline 5 & $5.33700 e-02 \times h^{2}$ & $2.8789 e-02 \times h$ & $1.4518 e+00 \times h^{2}$ & $1.9115 e-02 \times h$ \\
\hline 6 & $4.9291 e-02 \times h^{2}$ & $6.5325 e-01 \times h^{2}$ & $1.8996 e+00 \times h^{2}$ & $8.7364 e-01 \times h^{2}$ \\
\hline 7 & $4.6771 e-02 \times h^{2}$ & $1.3400 e-03 \times h$ & $1.0810 e+00 \times h^{2}$ & $1.2636 e-03 \times h$ \\
\hline 8 & $4.5462 e-02 \times h^{2}$ & $7.2842 e-01 \times h^{2}$ & $1.2577 e+00 \times h^{2}$ & $9.2338 e-00 \times h^{2}$ \\
\hline 9 & $4.4681 e-02 \times h^{2}$ & $1.5197 e-03 \times h$ & $8.8635 e-01 \times h^{2}$ & $3.6756 e-03 \times h$ \\
\hline
\end{tabular}

Table 3. The maximal errors of area-weighted averaging

\begin{tabular}{|c|c|c|c|c|}
\hline $\mathrm{n}$ & $e_{3}\left(F_{1}, n\right)$ & $e_{3}\left(F_{2}, n\right)$ & $e_{3}\left(F_{3}, n\right)$ & $e_{3}\left(F_{4}, n\right)$ \\
\hline 3 & $1.7281 e-02 \times h$ & $8.2961 e-01 \times h$ & $1.7331 e+00 \times h^{2}$ & $7.1663 e-01 \times h$ \\
\hline 4 & $8.4120 e-02 \times h^{2}$ & $4.3854 e-01 \times h^{2}$ & $1.1554 e+00 \times h^{2}$ & $6.1402 e-01 \times h^{2}$ \\
\hline 5 & $7.3895 e-02 \times h^{2}$ & $8.0248 e-01 \times h^{2}$ & $1.7331 e+00 \times h^{2}$ & $7.5509 e-01 \times h^{2}$ \\
\hline 6 & $6.8800 e-02 \times h^{2}$ & $8.5412 e-01 \times h^{2}$ & $1.7331 e+00 \times h^{2}$ & $8.1070 e-01 \times h^{2}$ \\
\hline 7 & $6.7316 e-02 \times h^{2}$ & $8.9488 e-01 \times h^{2}$ & $1.7331 e+00 \times h^{2}$ & $8.4060 e-01 \times h^{2}$ \\
\hline 8 & $6.6484 e-02 \times h^{2}$ & $9.2511 e-01 \times h^{2}$ & $1.7331 e+00 \times h^{2}$ & $8.6749 e-01 \times h^{2}$ \\
\hline 9 & $6.4620 e-02 \times h^{2}$ & $9.4713 e-01 \times h^{2}$ & $1.7331 e+00 \times h^{2}$ & $8.8737 e-01 \times h^{2}$ \\
\hline
\end{tabular}

In the following, we shall give a sufficient condition for the convergence in rate $O\left(h^{2}\right)$.

Theorem 1. Let $p_{0}$ be a vertex of $M$ with valence $n$, and $p_{i}, i=1, \cdots, n$ be its neighbor vertices. Suppose $p_{0}$ and $p_{i}, i=1, \cdots, n$ are on a sufficiently smooth regular parametric surface $\mathbf{S}(x, y) \in \mathbf{R}^{3}$ and there exist $q_{0}, q_{i} \in \mathbf{R}^{2}$ such that $p_{0}=\mathbf{S}\left(q_{0}\right), p_{i}=\mathbf{S}\left(q_{i}\right)$. Then in the following two cases

(1). $n=2 m, m>1, q_{i+m}=q_{0}-\left(q_{i}-q_{0}\right), \sum_{i=1}^{m} \lambda_{i}=1, \lambda_{i+m}=\lambda_{i}$,

(2). $n=2 m+1, m>1, \angle q_{i} q_{0} q_{i+1}=\frac{2 \pi}{2 m+1},\left\|q_{i}-q_{0}\right\|=\left\|q_{i+1}-q_{0}\right\|, \sum_{i=1}^{m} \lambda_{i}=1$, $\lambda_{i+1}=\lambda_{i}$,

$\sum_{i=1}^{n} \lambda_{i} \overline{\mathbf{n}}_{i, i+1}$ approximates the unit normal of the surface at the point $p_{0}$ to the accuracy $O\left(h^{2}\right)$.

Proof. Without loss of generality, we may assume $q_{0}=(0,0)$ and $q_{i}=\left(r_{i} \cos \left(\theta_{i}\right) h\right.$, $\left.r_{i} \sin \left(\theta_{i}\right) h\right)$. Since $\mathbf{S}(x, y)$ is a regular surface, we can use the notations and formulas proposed in Section 2.

It follows from (2) that,

$$
\overline{\mathbf{n}}_{i, i+1}=\overline{\mathbf{n}}_{0}\left(1-\frac{A_{i}\left(y_{u} z_{v}-y_{v} z_{u}\right) h-B_{i}\left(x_{u} z_{v}-x_{v} z_{u}\right) h+C_{i}\left(x_{u} y_{v}-x_{v} y_{u}\right) h}{\sin \left(\theta_{i+1}-\theta_{i}\right) r_{i} r_{i+1}}+O\left(h^{2}\right)\right) .
$$

Consider $\sum_{i=1}^{n} \lambda_{i} \overline{\mathbf{n}}_{i, i+1}$. By the explicit formulation of $\overline{\mathbf{n}}_{i, i+1}$, to prove the theorem,we merely need to prove

$$
\sum_{i=1}^{n} \lambda_{i} \frac{A_{i}}{\left(\sin \theta_{i+1}-\theta_{i}\right) r_{i} r_{i+1}}=0, \quad \sum_{i=1}^{n} \lambda_{i} \frac{B_{i}}{\left(\sin \theta_{i+1}-\theta_{i}\right) r_{i} r_{i+1}}=0,
$$




$$
\sum_{i=1}^{n} \lambda_{i} \frac{C_{i}}{\left(\sin \theta_{i+1}-\theta_{i}\right) r_{i} r_{i+1}}=0 .
$$

Firstly, we consider the case where $n=2 m$. Since $q_{i+m}=q_{0}-\left(q_{i}-q_{0}\right)$, we have $\theta_{i+m}=\pi+\theta_{i}, r_{i+m}=r_{i}$. Hence,

$$
\begin{aligned}
& \sum_{i=1}^{n} \lambda_{i} \frac{r_{i+1} \cos ^{2} \theta_{i+1} \sin \theta_{i}}{\sin \left(\theta_{i+1}-\theta_{i}\right)} \\
= & \sum_{i=1}^{m} \lambda_{i} \frac{r_{i+1} \cos ^{2} \theta_{i+1} \sin \theta_{i}}{\sin \left(\theta_{i+1}-\theta_{i}\right)}+\sum_{i=m+1}^{n} \lambda_{i-m} \frac{r_{i+1-m} \cos ^{2}\left(\pi+\theta_{i+1-m}\right) \sin \left(\pi+\theta_{i-m}\right)}{\sin \left(\theta_{i+1-m}-\theta_{i-m}\right)} \\
= & \sum_{i=1}^{m} \lambda_{i} \frac{r_{i+1} \cos ^{2} \theta_{i+1} \sin \theta_{i}}{\sin \left(\theta_{i+1}-\theta_{i}\right)}+\sum_{i=1}^{m} \lambda_{i} \frac{-r_{i+1} \cos ^{2} \theta_{i+1} \sin \theta_{i}}{\sin \left(\theta_{i+1}-\theta_{i}\right)} \\
\equiv & 0 .
\end{aligned}
$$

Using similar method, $\sum_{i=1}^{n} \lambda_{i} \frac{A_{i}}{\left(\sin \theta_{i+1}-\theta_{i}\right) r_{i} r_{i+1}} \equiv 0$.

Secondly, we consider the case where $n=2 m+1$. In this case, $\lambda_{i}, r_{i}$ and $\theta_{i+1}-\theta_{i}$ are all constant. Hence, to prove $\sum_{i=1}^{n} \lambda_{i} \frac{A_{i}}{\left(\sin \theta_{i+1}-\theta_{i}\right) r_{i} r_{i+1}} \equiv 0$, we only need prove $\sum_{i=1}^{n} \cos ^{2} \theta_{i} \sin \theta_{i+1}=0, \sum_{i=1}^{n} \cos ^{2} \theta_{i} \cos \theta_{i+1}=0, \sum_{i=1}^{n} \cos \theta_{i} \sin \theta_{i}$ $\sin \theta_{i+1}=0, \sum_{i=1}^{n} \cos \theta_{i} \sin \theta_{i} \cos \theta_{i+1}=0, \sum_{i=1}^{n} \cos \theta_{i+1} \sin ^{2} \theta_{i}=0$, and $\sum_{i=1}^{n} \sin \theta_{i+1} \sin ^{2} \theta_{i}=0$. We only prove one equation, with the proof of other equations being similar. Consider

$$
\begin{aligned}
\sum_{i=1}^{n} \cos ^{2} \theta_{i} \sin \theta_{i+1} & =\sum_{i=0}^{2 m} \cos ^{2} \frac{i-1}{2 m+1} 2 \pi \sin \frac{i}{2 m+1} 2 \pi \\
& =2 \sum_{i=1}^{2 m} \sin \frac{i}{2 m+1} 2 \pi \cos \frac{2(i-1)}{2 m+1} 2 \pi-\sum_{i=1}^{2 m} \sin \frac{i}{2 m+1} 2 \pi .
\end{aligned}
$$

Using the equality $\sum_{k=1}^{2 m} \sin \left(a_{0}+k d\right)=\frac{\cos \left(d / 2+a_{0}\right)-\cos \left(a_{0}+2 m d+d / 2\right)}{2 \sin d / 2}$, we have

$$
\begin{aligned}
& 2 \sum_{i=1}^{2 m} \sin \frac{i}{2 m+1} 2 \pi \cos \frac{2(i-11)}{2 m+1} 2 \pi-\sum_{i=1}^{2 m} \sin \frac{i}{2 m+1} 2 \pi \\
& \quad=\sum_{i=1}^{2 m}\left(\sin \frac{3 i-2}{2 m+1} 2 \pi+\sin \frac{-i+2}{2 m+1} 2 \pi\right)-\sum_{i=1}^{2 m} \sin \frac{i}{2 m+1} 2 \pi \\
& \quad=0 .
\end{aligned}
$$

Using the similar derivation above, we can prove $\sum_{i=1}^{n} \lambda_{i} \frac{B_{i}}{\left(\sin \theta_{i+1}-\theta_{i}\right) r_{i} r_{i+1}}=0$ and $\sum_{i=1}^{n} \lambda_{i} \frac{C_{i}}{\left(\sin \theta_{i+1}-\theta_{i}\right) r_{i} r_{i+1}}=0$.

Hence, under the condition (1) or (2), $\sum_{i=1}^{n} \lambda_{i} \overline{\mathbf{n}}_{i, i+1}$ has quadratic convergence rate. The theorem is proved. 
Corollary 1. Under the conditions of Theorem 1, if the weight $\lambda_{i}$ is defined as the arithmetic mean or the area-weighted averaging $\sum_{i=1}^{n} \lambda_{i} \overline{\mathbf{n}}_{i, i+1}$ approximates the unit normal of the surface at the point $p_{0}$ to accuracy $O\left(h^{2}\right)$.

Proof. When $\lambda_{i}$ is selected as arithmetic mean, $\lambda_{i}=\frac{1}{n}$. Obviously, in this case $\lambda_{i}=\lambda_{j}, \forall i, j$. By Theorem 1, the Corollary holds, when $\lambda_{i}$ is defined as the arithmetic mean. Denote the area of $\triangle p_{i} p_{0} p_{i+1}$ as $A\left(p_{i} p_{0} p_{i+1}\right)$. Then, we have $A\left(p_{i} p_{0} p_{i+1}\right)=\frac{1}{2}\left\|\mathbf{n}_{i, i+1}\right\|$. Under the condition of Theorem 1, it is easy to see that the coefficient of $h$ in $\sum_{i=1}^{n} A\left(p_{i} p_{0} p_{i+1}\right)$ and $\sum_{i=1}^{n} \mathbf{n}_{i, i+1}$ is cancelled. The Corollary holds.

Corollary 2. Under the condition (1) of Theorem 1, if the weight $\lambda_{i}$ is defined as the angle-weighted averaging $\sum_{i=1}^{n} \lambda_{i} \overline{\mathbf{n}}_{i, i+1}$ approximates the unit normal of the surface at the point $p_{0}$ to accuracy $O\left(h^{2}\right)$.

Proof. Let $\theta_{i, i+1}$ be the planar angle $p_{i} p_{0} p_{i+1}$ and let it be positive by convention. Then we can derive, $\theta_{i, i+1}=\theta_{i+1}-\theta_{i}+a^{(i)} h+O\left(h^{2}\right)$. Under the condition (1) of Theorem 1 $a^{(i+m)}=-a^{(i)}$. Hence, it is easy to see that the coefficient of $h$ in $\lambda_{i} \overline{\mathbf{n}}_{i, i+1}$ is cancelled. The Corollary is proved.

Remark 1. The convergence results are established under particular conditions. As pointed out in [11], these special cases are very useful and important. A number of numerical simulations of geometric partial differential equations are conducted over a triangulated domain formed by a uniform three-directional or four-directional partition. Both partitions satisfy the condition in Theorem 1 .

Remark 2. An interesting observation is that the condition of the discretization scheme of normals having quadratic convergence is exactly the same as the condition of a discretization of gradient having quadratic convergence proposed in [11]. (Theorem 4.1 in [11] presents only the condition (1). In fact, by using similar method with [11], under the condition (2), the discretization of gradient has also quadratic convergence. )

\section{Counterexamples to Convergence of Curvature and Normals}

In the previous section, we have studied the convergence of the discrete unit normal. The convergence property of the discrete Gaussian curvature and mean curvature has been considered in [9, [10, 11] and [5. But none of discretization schemes has been proved to be convergent over any non-degenerate triangle surfaces. A natural questions is raised: can one build a discretization scheme of Gaussian curvature and mean curvature converging over any non-degenerate triangle surfaces? In this section, by a counterexample we shall give a negative answer for the question.

Let $p_{0}$ be a vertex of $M$ where the Gaussian curvature is are to be approximated and $p_{i}, i=1, \cdots, n$ be its neighbor vertices. We make a hypothesis 
that the discretization scheme of Gaussian curvature involving one-ring neighbor vertices of $p_{0}$, denoted as $H\left(M, p_{0} ; p_{1}, \cdots, p_{n}\right)$, is convergent for any triangle mesh surface $M$. Suppose $M$ is a given triangle surface approximating the surface $\mathbf{S}(x, y)=(x, y, f(x, y))^{T}, f(x, y)=B_{02} x^{2}+B_{11} x y+B_{02} y^{2}$ and the origin $p_{0}=(0,0,0)$ is a vertex of $M$. Assume the valence of the origin point is 4 and the neighbor points are $p_{i}=\mathbf{S}\left(\mathbf{q}_{i}\right), i=1, \cdots, 4$, where $\mathbf{q}_{1}=h(1,2), \mathbf{q}_{2}=$ $h(-1,-2), \mathbf{q}_{3}=h(-1,2)$ and $\mathbf{q}_{4}=h(1,-2)$ (see fig.1.a). Since Gaussian curvature of $\mathbf{S}(x, y, z)$ at $p_{0}$ equals to $4 B_{02} B_{20}-B_{11}^{2}$, by the convergence property of $H\left(M, p_{0} ; p_{1}, \cdots, p_{n}\right)$, we have $\lim _{h \rightarrow 0} H\left(M, p_{0} ; p_{1}, p_{2}, p_{3}, p_{4}\right)=4 B_{02} B_{20}-B_{11}^{2}$.

Suppose $\widehat{M}$ is another given mesh surface approximating the surface $\widehat{\mathbf{S}}(x, y)=$ $(x, y, \widehat{f}(x, y))^{T}, \widehat{f}(x, y)=\left(4 B_{02}+B_{20}\right) x^{2}+B_{11} x y$, and the origin $\mathbf{O}:(0,0,0)$ is a vertex of $\widehat{M}$ where the curvature needs to be approximated. The neighbor points of the origin are $\widehat{p}_{i}=\widehat{\mathbf{S}}\left(\mathbf{q}_{i}\right), i=1, \cdots, 4$, where $\mathbf{q}_{1}=h(1,2), \mathbf{q}_{2}=$ $h(-1,-2), \mathbf{q}_{3}=h(-1,2)$ and $\mathbf{q}_{4}=h(1,-2)$. The Gaussian curvature of $\widehat{\mathbf{S}}$ at $\mathbf{O}$ is $-B_{11}^{2}$. By the convergence property of $H$, we have $\lim _{h \rightarrow 0} H\left(\widehat{M}, p_{0} ; \widehat{p}_{1}, \widehat{p}_{2}\right.$, $\left.\widehat{p}_{3}, \widehat{p}_{4}\right)=-B_{11}^{2}$. Obviously, by the formulation of $f(x, y)$ and $\widehat{f}(x, y)$, for any $h$, $p_{i}=\widehat{p}_{i}, i=1, \cdots, 4$. Since the discretization scheme $H$ merely involves one-ring neighbor vertices of $p_{0}$, by $p_{i}=\widehat{p}_{i}, H\left(\widehat{M}, p_{0} ; \widehat{p}_{1}, \widehat{p}_{2}, \widehat{p}_{3}, \widehat{p}_{4}\right)=H\left(M, p_{0} ; p_{1}, p_{2}, p_{3}, p_{4}\right)$ for any $h$. Hence, $\lim _{h \rightarrow 0} H\left(\widehat{M}, p_{0} ; \widehat{p}_{1}, \widehat{p}_{2}, \widehat{p}_{3}, \widehat{p}_{4}\right)=\lim _{h \rightarrow 0} H\left(M, p_{0} ; p_{1}, p_{2}, p_{3}, p_{4}\right)$. But $-B_{11}^{2}$ is not always equal to $4 B_{02} B_{20}-B_{11}^{2}$. Therefore, a contradiction appears. So, the hypothesis with $H\left(M, p_{0} ; p_{1}, \cdots, p_{n}\right)$ being convergent for any mesh surface does not hold.

Since the mean curvature of $\mathbf{S}(x, y)$ and $\widehat{\mathbf{S}}(x, y)$ at the origin equals to $B_{02}+$ $B_{20}$ and $4 B_{02}+B_{20}$ respectively, we can show that one can not construct a discretization scheme of mean curvature converging over any mesh surface by using the similar method with the above,

From above, if merely using one-ring vertices, we can not build discretization schemes of Gaussian curvature and mean curvature converging over any mesh surface. For fixed integer $k$, using $k$-ring vertices, can we construct a

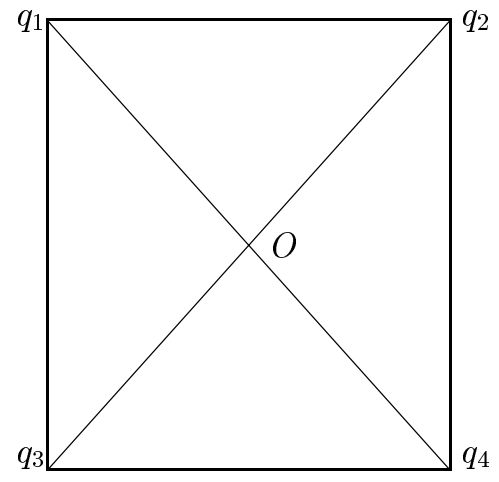

a

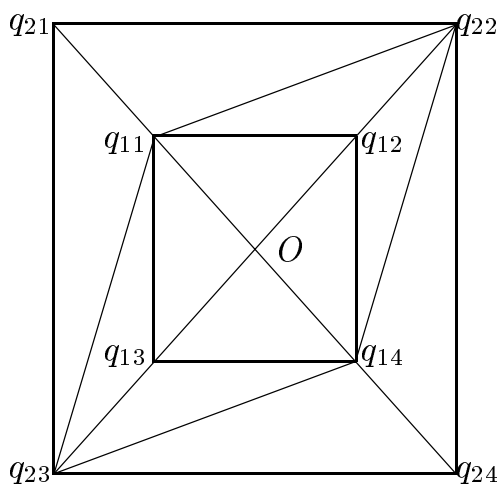

b

Fig. 1. A counterexample to convergence of curvature and normal 
discretization scheme of Gaussian and mean curvature converging over any mesh surface?

Suppose the $j$-th ring vertices around $p_{0}$ is $p_{j, i}=\mathbf{S}\left(\mathbf{q}_{j, i}\right)$, where $j \leq k, \mathbf{q}_{j, i}=$ $j \mathbf{q}_{i}, i=1, \cdots, 4$ (Fig.1.b shows the case where $k=2$ ). Obviously, for any $h$, $S\left(\mathbf{q}_{j, i}\right)=\widehat{S}\left(\mathbf{q}_{j, i}\right), j \leq k, i=1, \cdots, 4$. Hence, by using similar method with the above, we can show that for fixed integer $k$, if only use $k$-ring vertices, we can not build discretization schemes of mean curvature converging over any mesh surface. It is well known that Laplace-Beltrami operators relates closely to the mean curvature normal. Let $p$ be a surface point on two-dimensional manifold $\mathcal{M}$. Then $\left\|\Delta_{\mathcal{M}} p\right\|=2 H(p)$, where $\Delta_{\mathcal{M} p}$ is the Laplace-Beltrami operator and $H(p)$ is the mean curvature at $p$. Hence, by above results, for fixed integer $k$, if only $k$-ring vertices are used, we can not build discretization schemes of LaplaceBeltrami operators converging over any mesh surface.

In [5], a open question is raised: Find a linear combination of the normals of the triangular faces, based on geometric considerations, that approximates the normal of the surface to $O\left(h^{2}\right)$. We shall give a negative answer for the open question. Suppose that the vertexes $\mathbf{S}\left(q_{1 i}\right), i=1, \cdots, 4$ (see Fig.1.b) are the vertexes of $M$ where the unit normals are to be approximated. We make a hypothesis that there exits a linear combination of the normal of the triangular faces that approximates the normal at $\mathbf{S}\left(q_{1 i}\right)$ to $O\left(h^{2}\right)$. By Lemma 1] under the hypothesis, we can build a discretization schemes involving 2-ring vertexes which approximates the Gaussian curvature at $\mathbf{O}$ to accuracy $O(h)$. The conclusion contradicts to the above results. Hence, the hypothesis does not hold i.e. for any mesh surface, one can not find a linear combination of the normals of the triangular faces that approximates the normal of the surface to $O\left(h^{2}\right)$.

Remark 3. In this counterexample, the valence of $p_{0}$ and $p_{11}$ is 4 and 6 respectively. In fact, by using similar method, we can show one can not build a discretization schemes of Gauss and mean curvature which convergent at $p_{11}$. Hence, even if the valence of vertexes in mesh surface is bigger than 4, we also can not build convergent discretization schemes of Gauss and mean curvature for any mesh surface.

Remark 4. The points $p_{i}$ or $p_{j i}$ in this counterexample are under-determined for quadratic fit. As pointed out in [5] and [11], if the quadratic fit method has a unique solution, one can approximate the Gaussian curvature and mean curvature to accuracy $O(h)$ and the unit normal to accuracy $O\left(h^{2}\right)$. Then, when the quadratic fit method has a unique solution, can one find a convergence discretization scheme of Gaussian and mean curvature depending only on edge length, angles, and areas of triangle faces? We conjecture that the answer to the question is negative. If the conjecture holds, quadratic fit methods can be the most general method for finding convergent discretization schemes for geometry operators.

Acknowledgements. The work of the first author is supported by NSFC under grant 10401021 and China Postdoctoral Science Foundation. The work of the second author is supported in part by NSFC under grant 10371130, National 973 Project under grant 2004CB318006. 


\section{References}

1. M. Desbrun, M.Meyer, P.Schröder and A.H.Barr, Implicit fairing of irregular meshes using diffusion and curvature flow, SIGGRAPH99, 317-324,1999.

2. G H. Liu, Y.S. Wong, Y.F.Zhang, H. T Loh, Adaptive fairing of digitized point data with discrete curvature, Computer Aided Design, 34 (4), 309-320, 2002.

3. Jean-Louis Maltret, Marc Daniel, Discrete curvatures and applications : a survey, preprint, 2003.

4. U. F. Mayer, Numerical solutions for the surface diffusion flow in three space dimensions. Computational and Applied Mathematics (to appear),2001.

5. D.S. Meek, D.J. Walton, On surface normal and Gaussian curvature approximations given data sampled from a smooth surface, Computer Aided Geometric Design, 17 (2000) 521-543.

6. M. Meyer, M. Desbrun, P. Schroder, A. Barr, Discrete differential-geometry operator for triangulated 2-manifolds, in:Proc. VisMath'02, Berlin, Germany.

7. G. Taubin, A signal processing approach to fair surface design, in SIGGRAPH'95 Proceedings 351-385.

8. C. Wollmann, Estimation of principal curvatures of approximated surfaces, Computer Aided Geometric Design,vol 17, 621-630, 2000.

9. G. Xu, Convergence analysis of a discretization scheme for Gaussian curvature over triangular surfaces, submitted for publication.

10. G. Xu, Convergence of discrete Laplace-Beltrami operator over surfaces, Computers and Mathematics with Applications, 48 (2004), 347-360.

11. G. $\mathrm{Xu}$, Discrete Laplace-Beltrami operators and their convergence, Computer Aided Geometric Design 21 (2004)767-784. 